\title{
La enseñanza del español en Marruecos: del pasado al presente
}

\section{ZINEB BENYAYA}

Facultad de Filosofía y Letras, Departamento de Lengua Española

Grupo de Investigación: GILA (Grupo de Investigación de Lingüística Aplicada)

Recibido: 5 Julio 2006 / Aceptado: 12 septiembre 2006

ISSN: $1697-7467$

\begin{abstract}
RESUMEN: Este artículo trata de realizar un recorrido por la historia del español en Marruecos, desde el momento en que se introduce con los primeros misioneros franciscanos, pasando por el Protectorado, en que se convierte en primera lengua oficial instaurada por el colonizador, hasta llegar a la situación actual, para contemplar, en medio de las vicisitudes políticas, qué puesto ostenta dentro del panorama de enseñanza de idiomas y en el seno del sistema educativo marroquí.
\end{abstract}

Palabras clave: Enseñanza del español, Marruecos

\begin{abstract}
The aim of this article is to make a route by the history of Spanish language in Morocco, Since the moment which it gets up by the first Franciscan Missionaries and their Protectorate, in which it became the first official language restored by the colonist, until the current situation., in the central of the political vicissitudes we tried to contemplate, what kinds of positions shows within the panorama of languages learning and in the Moroccan education system.
\end{abstract}

Key words: Spanish teaching, Morocco

\section{INTRODUCCIÓN}

Mencionar la historia compartida, la cercanía geográfica o los lazos lingüísticos y culturales que unen España y Marruecos se ha convertido casi en un tópico y en una retórica carente de sentido, en cualquier reunión oficial, congreso o manifestación cultural que terminan, casi siempre, de la misma forma que empiezan: con un mutuo y perenne desconocimiento entre los vecinos de las dos orillas del Mediterráneo. Lo cierto es que, efectivamente, un recorrido por la historia de ambos países deja traslucirse una profunda ósmosis que hubo de engendrar múltiples intercambios y traducirse en herencias a uno y otro lado del Estrecho que separa físicamente España de Marruecos.

Las palabras que dominan el léxico analítico de la prensa, tanto española como marroquí, describen la relación hispano-marroquí en los términos de amor/odio, convivencia/confrontación y coinciden en hablar de malentendidos, resentimientos, incompatibilidades, incomprensión recíproca, reticencias etc., visión que plasma, con bastante transparencia, la situación de nuestras relaciones bilaterales, con todos los hitos que jalonan una relación de 
vecindad, y con todo lo que ello pueda tener de trascendencia en sectores como el de la cooperación cultural o el de la enseñanza del español que es el tema que, particularmente, nos preocupa en esta investigación.

\section{UN RECORRIDO POR LA HISTORIA}

Estimamos conveniente, para contemplar qué puesto ostenta el español dentro del panorama de enseñanza de idiomas en Marruecos y, en especial, en el seno del sistema educativo del país, realizar una diacronía de los hechos que incidieron, de la forma más relevante, en el desarrollo o evolución de esta enseñanza en Marruecos, hasta llegar a la situación actual.

\section{1. Época precolonial}

Según Lourido Díaz, ${ }^{1}$ a lo largo de la historia, entre la Europa medieval y el Islam se han dado constantemente conflictos bélicos y batallas justificadas desde la fe cristiana -y musulmana, sin duda-, pero lo cierto es que, tras el fracaso de las Cruzadas, más bien se libraron batallas "apostólicas y apologéticas". Esta acción sería conducida por religiosos cristianos que se armarían -en la medida que les fuera posible- de profundo conocimiento sobre el Islam. Los famosos Studia linguarum $^{2}$ respondían a esa necesidad de conocer el Islam para poder tender puentes de comprensión y entablar diálogo con los musulmanes.

En tiempos posteriores, correspondientes ya a la época precolonial en Marruecos, la labor cultural es atribuible todavía a la Iglesia, si bien hay que señalar que, en el momento de su institucionalización en Marruecos, la labor de la Iglesia no destaca precisamente por su relevancia, pero es a partir de ese momento cuando que comienza una tímida tarea de culturización que cristaliza en la aparición de las primeras escuelas dirigidas por las misiones franciscanas, las cuales estaban destinadas exclusivamente a cristianos extranjeros, si bien irían incorporando progresivamente a alumnos judíos y musulmanes. Esta labor de los religiosos cristianos se extiende hasta bien entrado el siglo XX y se acentúa de forma especialmente notoria en la época colonial. Lourido Díaz menciona especialmente al padre Lerchundi que considera como el propulsor de la cultura española en Marruecos, y es a quien se debe la reforma y modernización de las escuelas de las misiones, la creación en Tetuán de la Escuela para la Enseñanza del Árabe, además de una de las primeras imprentas establecidas en Marruecos y de varios proyectos que no llegaron a buen término ${ }^{3}$.

\section{2. Época colonial}

La época colonial registra una entusiasta labor cultural que Valderrama no duda en calificar de "una de las más bellas realidades labradas amorosamente por España en Marrue-

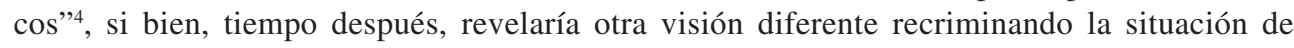

${ }^{1}$ Lourido Díaz (1993:54).

${ }^{2}$ Escuelas especializadas en el estudio de la lengua árabe, de la teología y filosofía islámica.

${ }^{3}$ Lourido Díaz (1993: 65).

${ }^{4}$ Valderrama Martínez (1954:31) 
desorganización característica del sistema de enseñanza regido por el Protectorado español en Marruecos ${ }^{5}$. El español, con el Protectorado, se convierte en la primera lengua impuesta por el colonizador tanto en la administración como en las instituciones, a la vez que se establece como segunda lengua hablada por los nativos que tenían que establecer sus vínculos sociales y comerciales con los españoles, aunque, cabe señalar que, en este ámbito, no se trataba de ninguna política predeterminada llevada a cabo con el sistemático afán de enseñar el idioma de los conquistadores a la población marroquí, sino que, más bien, se produjo una difusión natural, debido al progresivo contacto de la población marroquí con la colonia española ${ }^{6}$. Las escuelas, tanto de primaria como de secundaria, distribuidas por las distintas ciudades de la Zona y por los núcleos de concentración de la población española, eran regidas por la Administración Española, quien velaba por esta enseñanza dedicada específicamente a la población española afianzada en Marruecos -aunque, a esta enseñanza, también tenía acceso la población marroquí que se incorporaría en muy menor medida ${ }^{7}$-. Al lado de ésta, coexistía una enseñanza -la única, de hecho, conocida antes del Protectorado- de corte tradicional, articulada en torno a los msaed o mezquitas $^{8}$, dedicada a los niños musulmanes, basada en la enseñanza literal del Corán que culmina con el grado de taleb o'faqih y que faculta al que lo posee a oficiar ceremonias religiosas y a impartir la misma enseñanza legada, y una vez más, de la misma manera que se le ha legado.

Esta enseñanza es mantenida en su esencia por la Administración Española ${ }^{9}$, si bien es modificada en la forma y en lo que a organización se refiere ${ }^{10}$ : se llegan a redactar programas y planes de estudio y se decretan leyes que contemplan el futuro de los titulados en esta enseñanza religiosa, y paulatinamente, se va abandonando la mezquita como lugar de instrucción para darse en lugares concebidos especialmente para esta tarea. Además se desarrolla un nuevo tipo de enseñanza marroquí -esta vez moderna-, de gestión nacional, pero bajo la supervisión de España, en la que la instrucción general se realizaba en árabe, eso sí, con el español como asignatura fundamental, y con la misma categoría que la lengua árabe, tanto en las escuelas de primarias como en la secundaria, y es esta nueva enseñanza la que va ganando cada vez más confianza, agrado y adeptos entre la población marroquí ${ }^{11}$.

\section{3. Época postcolonial}

En un Marruecos independiente, la enseñanza se va nacionalizando, y el Gobierno Marroquí toma las riendas de todos los sectores que han estado en manos del colonizador, y el de la

\footnotetext{
${ }^{5}$ Véase Valderrama Martínez, Historia de la acción cultural de España en Marruecos (1912-1956) Alta Comisaría de España en Marruecos, Editora Marroquí, 1956, Tetuán.

${ }^{6}$ Manuela Marín Acción colonial y comunicación lingüística en Marruecos en http://cvc.cervantes.es/ obref/anuario/anuario_03/munoz/p03.htm.

${ }^{7}$ Véase Ricardo Ruiz Orsatti, La enseñanza en Marruecos, Tetuán, La Papelera Africana, 1918.

${ }^{8}$ Véase Amina Loh (1968: 11) y Cf. Salas Larrazábal (1992: 237).

9 Valderrama Martínez (1954: 31-36).

${ }^{10}$ El español se introduce por vez primera en el sistema educativo marroquí en el año 1936, con la inauguración del Instituto de Libre enseñanza de Tetuán. Tomo el dato de Tanouti./ Muati (1997: 41).

${ }^{11}$ Para una visión exhaustiva sobre el desarrollo de la enseñanza en la época del Protectorado español en Marruecos, véase Tomás García Figueras, Notas sobre instrucción y cultura en Marruecos (hasta 1935), Alta comisaría de España en Marruecos, 1940, Tetuán. Para una visión más sintetizada, véase Fartakh (1997: 5963).
} 
enseñanza -no sin dificultades- se adapta a la política de marroquinización que cursa el país recién emancipado ${ }^{12}$.

La acción cultural española en Marruecos en el marco de la independencia, empieza a cristalizar en medio de una vorágine de altibajos y vicisitudes políticas que vienen a condicionarla en gran medida. Sin embargo, hay que decir que consigue abrirse camino y, es curioso constatar cómo justamente en ese momento en que empieza a disminuir la presencia de la colonia española, paradójicamente, empieza a percibirse un interés por fomentar esa acción cultural. Aunque, es un interés que responde, más bien, a una política exterior que ahora pretende ser expansiva -culturalmente hablando- bien para encarar la política de un Gobierno Marroquí que viene accionando un riguroso plan de marroquinización -con la consiguiente arabización del sector de enseñanza-, o a la competencia del francés que ha venido a instaurarse como la segunda lengua oficial, y la de la administración por excelencia, frente a una pasmosa discriminación del español en todos los sectores, incluso en sus -recientemente perdidas-, zonas de influencia, en que gozaba de supremacía. Desaparecen, en consecuencia, algunos centros y se cierran todas las librerías españolas o se dedican a la venta de libros en árabe y francés.

España, a raíz de esta situación, se percata de la urgencia de una política cultural en Marruecos, y este empeño se traduce en la reorganización de sus estructuras educativas, además de la creación de nuevos centros e instalaciones como la Oficina Cultural en 1963 y los Centros Culturales de Fez y Rabat en $1971^{13}$.

\subsubsection{Los años setenta}

El Convenio Cultural Hispano-Marroquí de 7 de julio de 1957 venía a ser un intento de limar las asperezas y abrir una nueva página en la historia de España y Marruecos. Se pretendía, así, olvidar el reciente pasado de subordinación e iniciar una etapa de cooperación y "verdadera amistad", si bien los frutos tardaron en brotar, y hubo que esperar a la época democrática para poder palpar cambios significativos en el escenario de la cooperación cultural y de las relaciones entre ambos países. Por una parte, el resentimiento por el pasado reciente, sin duda, no hizo ver con buenos ojos la iniciativa española en Marruecos, y por otra la incomprensión y el empecinamiento en un "diálogo de sordos", durante mucho tiempo, no benefició a ninguna de las dos partes. A propósito de esta situación, se pronuncia Moratinos:

La sociedad española no cesó, desde 1912 hasta nuestra salida poco airosa del Sáhara Occidental, de configurar su relación con los "moros" más que en los términos de enfrentamiento, de malentendidos y de ocasiones perdidas. ${ }^{14}$

Sin embargo, -sigue argumentando Moratinos-:

La responsabilidad no fue exclusivamente española, sino que las autoridades de Rabat, por estrictas razones políticas, aplicaron a rajatabla el bilingüismo oficial:

${ }^{12}$ El Mejdoubi (1999: 42).

${ }_{13}$ Morales Lezcano (1993: 164).

${ }^{14}$ Moratinos (1993: 177). 
árabe y francés, mientras que nuestra lengua y cultura no sólo no encontraron apoyo, sino que hubieron de enfrentarse a todo tipo de dificultades para su difu$\operatorname{sión}^{15}$.

Fue un momento de apatía y de una presencia débil del español, en volumen y difusión, que se tradujo en un total desinterés y en una falta de motivación por el aprendizaje de ese idioma. Larbi Messari, ex ministro de Comunicación, señala cómo cuando el gobierno incitó a los alumnos en 1978 a optar por el español, como una alternativa más entre el inglés o el alemán, esta incitación encontraba reticencias reales ${ }^{16}$.

En los centros marroquíes, se venía utilizando un material didáctico arcaico e inadecuado, que conservaba todavía esa imagen trasnochada y tópica de la España "flamenca y torera". Eran manuales eminentemente de elaboración francesa, como el Sol y Sombra, Por el mundo hispánico, Lengua y vida, La Pratique de l'espagnol o el ya clásico ¿Qué tal Carmen?. Los derechos de autor, importante fuente de ingresos para muchos inspectores franceses, eran una razón añadida para ese cuasi monopolio de la enseñanza del español, y de la consecuente desatención a los textos españoles que, por otra parte, son todos muy recientes ${ }^{17}$.

\subsubsection{Los años ochenta}

En la perspectiva de los ochenta empiezan, muy lentamente, a vislumbrarse algunos cambios en el panorama de la enseñanza del español y a consolidarse, cada vez más, entre los responsables marroquíes la conciencia de la necesidad de un nuevo enfoque metodológico desligado del francés que, hasta entonces, había sido el modelo para la enseñanza del español. Así lo atestigua el texto siguiente, que pertenece al Programa de Español que trazó el Ministerio de Enseñanza Primaria y Secundaria en 1976:

La enseñanza del español no debe pasar por la relación con el francés. Es peligroso acostumbrar a los alumnos a traducir al francés en lugar de aprender a pensar únicamente en la lengua extranjera ${ }^{18}$.

Los años ochenta, pues, asisten a una relación más fluida y porosa que desemboca en una meditación y en un análisis de las deficiencias e inconveniencias de la política cultural hasta entonces llevada a cabo. En 1979, un nuevo convenio es firmado en aras de darle un impulso a la situación de anquilosamiento a la que había llegado la relación entre ambos países. Este último estaba especialmente enfocado hacia la enseñanza e investigación. En este sentido, resultan especialmente significativos, el artículo II de este convenio que estipula que:

15 Ibid.: p. 180.

16 Naji (2000: 158).

${ }^{17}$ El único manual de elaboración española que llegó a programarse en los institutos marroquíes fue el Para empezar, ya en los ochenta, mediante una donación de nueve mil ejemplares, efectuada por el Ministerio español de Información y Cultura. Véase Aziza Bennani (1996:286).

${ }^{18}$ Entiéndase aquí la española. Programme d’espagnol, instructions officielles. Ministère de 1’Enseignement Primaire et Secondaire, p.6, Setiembre 1976. 
Cada país se esforzará en facilitar la impresión de los manuales o textos escolares, especialmente los que se utilizan en los establecimientos de enseñanza secundaria para la enseñanza de disciplinas literarias e históricas. A tal efecto, se constituirán comisiones técnicas integradas por expertos de ambos países que procederán a dicha revisión y propondrán modificaciones que estimen justas y pertinentes.

y el artículo XI que apunta lo siguiente:

(...) ambos Gobiernos facilitarán el establecimiento y el funcionamiento en sus Facultades de Letras, Cátedras y puestos de lectores de la lengua y cultura del otro país. Los dos Gobiernos juzgan deseable para la eficacia de la enseñanza que, en la medida de lo posible, se confíen los puestos de lectores a profesores españoles en Marruecos y a profesores marroquíes en España. ${ }^{19}$

No obstante, este marco legal, aunque favorable, no fue suficiente como para dar el impulso definitivo a la cooperación cultural hispano-marroquí, ni siquiera contribuyó a suavizar las distintas dificultades que se imponían con insistencia ante la difusión del español. Problemas como la convalidación de títulos ${ }^{20}$, intercambio de lectores o la falta de circulación del libro español, quedaron en suspenso. Asimismo, En materia de enseñanza del español, la situación se encontraba todavía dominada por la dirección francesa, ya que la inspección se llevaba a cabo en coordinación con un Inspector francés que supervisaba esta enseñanza en los institutos marroquíes en total ausencia de España. Y -digámoslo así- todo quedó en un "buen convenio" que constituía, sin duda, un excelente cimiento para la edificación de una sólida cooperación, pero siempre y cuando una voluntad verdadera decidiera llevarlo a la práctica.

A. Bennani ${ }^{21}$, presidenta de la Asociación de Hispanistas Marroquíes, subraya que buena parte de la culpa de esta situación la tienen los prejuicios, y la falta de una visión valorizadora del otro, desligada del tópico. Asimismo, la vinculación de roces y conflictos -quizá- en el terreno político, totalmente ajenos a lo cultural, con el destino de la cooperación cultural es otra causa relevante, y trae a colación las palabras de Antonio Gala que, en cierta ocasión, diría:

¡Basta de sardinizar las relaciones hispano-marroquíes!

Todo ello tiñe, ineluctablemente de esterilidad e inercia las relaciones culturales hispano-marroquíes. Las declaraciones del rey Hassan II en el País en vísperas de su visita oficial a España, son especialmente significativas, y anuncian una especie de "reconciliación" con el español:

\footnotetext{
${ }^{19}$ Convenio de Cooperación Cultural entre el Gobierno De España y el Gobierno del reino de Marruecos, Madrid, 8 de noviembre de 1979.

${ }^{20}$ a causa de la inoperancia del convenio y al vacío legal consecuente, muchos de los titulados marroquíes en universidades españolas -excepto las de Madrid- tuvieron problemas a la hora de homologar sus títulos, lo cual hizo que muchos de los universitarios -actuales hispanistas- se replantearan la posibilidad de estudiar en España y optar por hacerlo en Francia. Véase Cecilia Fernández Suzor (1992: 329).

${ }^{21}$ Aziza Bennani (1996: 288).
} 
Es un error monumental no aprender más el idioma español en Marruecos ${ }^{22}$.

Toda esa voluntad demostrada por ambas partes por propulsar sus relaciones bilaterales, y que se ha dejado traslucir en más de una ocasión, es positiva. Sin embargo, el informe de la Oficina Cultural Española del año 1991 no es muy halagüeño ${ }^{23}$ y delata una ausencia y un desconocimiento de la cultura española en Marruecos, que en ocasiones -y según de qué región se trate- llega a ser absoluto, concretamente entre los sectores más activos de la sociedad, los cuales tienen como referencia el francés, y optan siempre por Francia, como es lógico, en el caso de decidir salir del marco de su país. Incluso -y el dato resulta paradójicola mayoría de los hispanistas marroquíes se han formado en universidades francesas, y han realizado sus tesis doctorales bajo la dirección de profesores franceses ${ }^{24}$.

Por otra parte, testimonios de profesionales de la enseñanza dejan patente la situación de aislamiento y desamparo en la que se encontraba el profesor de español en las aulas marroquíes. No se disponía de ningún material de apoyo didáctico que se adecuara a las necesidades de los alumnos y que facilitara la tarea de enseñar, y además, otro asunto no menos peliagudo, en el marco de esa situación, era el de convencer a esos alumnos de la utilidad que podía tener el aprendizaje de ese idioma en la perspectiva de un futuro. Todo ello contribuía a situar el español en clara desventaja con respecto a los otros idiomas. Situación que reflejaron muy bien las estadísticas del Ministerio de Educación Nacional del curso (1987-1988), frente a un 92\% que estudiaban inglés, sólo un 7,23\% de los alumnos estudiaban español, cifra que, a pesar de todo, superaba con creces la del alemán que sólo despertaba el interés de un $0,77 \%$ restante de los alumnos ${ }^{25}$.

\subsubsection{Los años noventa}

Ante esta situación, España es enfrentaba al desafío de reorganizar su misión educativa, con el propósito de readaptar sus dependencias, en lo que a sentido y función se refiere, e ir, a la vez, difundiendo de forma eficaz su lengua y cultura, en aras de conseguir su total afianzamiento y consolidación dentro el sistema educativo marroquí.

Para sobrepasar el bache y superar esta engorrosa situación, en 1991, se crea ex profeso una comisión interministerial, en la que se involucran el Ministerio de Asuntos Exteriores, el de Educación y el de Cultura. Esta comisión se replanteará, seriamente, la estrategia de la acción española en Marruecos, en el plano de lo cultural. En el mismo año, se crea -transforma- el mayor organismo responsable de la difusión de la lengua y cultura españolas, que es el Instituto Cervantes, que venía contando con la experiencia de sus "predecesores físicos" que eran, lógicamente, los Centros Culturales, pero ya con una maduración en el método y mayor profesionalización en la enseñanza del español, el cual va a emprender -en colaboración con la Consejería de Educación de la Embajada Española en Rabat- una acción de cooperación con los organismos responsables de la enseñanza del español en el sistema

\footnotetext{
${ }^{22}$ Entrevista del Rey Hassan II con El País, setiembre de1989.

${ }_{23}$ Véase Moratinos (1993: 181).

${ }^{24}$ Del Pino (1990: 258).

25 Tomo estos datos de Mustapha Chouiref (1991: 108).
} 
educativo marroquí ${ }^{26}$. Se inicia así una especie de enlace entre una Consejería de Educación que "propone" y un Ministerio de Educación marroquí que "dispone".

Este esfuerzo por fomentar su acción educativa, le supuso a España dedicar un generoso presupuesto a este proyecto, y que en 1995 llega a rondar los 1900 millones -de las entonces españolas pesetas-, en aras de difundir su lengua y cultura entre programas de becas, actividades culturales e intercambios entre las distintas universidades ${ }^{27}$.

Aunque la Comisión Mixta hispano-marroquí todavía revela el deseo de abordar una mejor reasignación de los fondos y estima que es escaso efecto para tan generosa política, la verdad es que el español en la última década ha conocido un gran despegue y ha comenzado a calar fuerte, empezando, gradualmente, a ganarle terreno a los otros idiomas que le hacen competencia dentro de la enseñanza secundaria marroquí.

\section{La eStructura del Sistema EdUCATIVo MaRroquí}

El sistema educativo marroquí, desde la enmienda de 1985, se estructura -de la siguiente manera- en una primera etapa correspondiente a la Enseñanza Preescolar, y es llevada a cabo en escuelas coránicas al estilo tradicional, aunque es preciso decir que las escuelas maternales y jardines de infancia, al estilo moderno, están muy extendidas.

La segunda etapa, corresponde a la Enseñanza Fundamental y consta, a su vez, de dos etapas: la primaria constituida por seis cursos, y la Preparatoria de tres, al cabo de los cuales se puede acceder o bien a la enseñanza secundaria, o a la Formación Profesional.

La enseñanza secundaria, con diferentes ramas, y constituida, por tres cursos igualmente, es la equivalente al anterior Bachillerato español, y es la que faculta, una vez superada, el acceso a los Estudios Superiores. En el 2002, esta estructura, aunque es mantenida en su esencia, comprende una leve remodelación que afecta, fundamentalmente, a las denominaciones, pasándose a llamar, el primer año de Bachillerato, como Ciclo de Cualificación (Tronco Común), mientras que los equivalentes a los anteriores cursos segundo y tercero, pasan a ser primero y segundo de Bachillerato, respectivamente.

La primera lengua extranjera, con carácter obligatorio es el francés, y empieza a impartirse a partir del segundo curso de la enseñanza primaria. La segunda lengua extranjera, sin embargo, no se incorpora hasta mucho más tarde, en la enseñanza secundaria, y el sistema ofrece la posibilidad de elegir entre varios idiomas, entre los cuales figura -compite- el español ${ }^{28}$.

El español, como lengua extranjera, se imparte en los tres niveles de secundaria y en todas sus ramas, y hasta el 2002, tenía establecida -en los tres cursos- una diferencia de horario semanal, según se trataba de la rama de Letras (4 horas), de la de Ciencias (3 horas) o de la de Letras originales (2 horas). A partir de esa fecha, la enseñanza del español, en el primer nivel, se unifica en un tronco común al que se le aplica el mismo programa con una única distribución horaria: 4 horas semanales para todas las ramas.

${ }^{26}$ Fernández Suzor (1993:169).

${ }^{27}$ Moratinos (1993: 186).

${ }^{28}$ Para un estudio exhaustivo sobre el sistema educativo marroquí, véase Jesús Romero López, Algunos retazos sobre la educación no universitaria en Marruecos, http://members.es.tripod.dekasbah01/ educacion_marroqui.htm. 
Algunos liceos, sin embargo, ofrecen la posibilidad de cursar un tipo de especialidad en lengua española, con más horas de español, cuyos alumnos suelen orientar su carrera hacia la de Filología Hispánica, aunque no con carácter exclusivo. Incluso se venía experimentando, desde el curso 1997-98, con una sección especial de lenguas, que en la correspondiente al español, denominada OLE (Opción de Lengua Española), contaría con un total de siete horas semanales ${ }^{29}$, si bien el proyecto, hasta el momento, sigue siendo exclusivo de unos pocos centros e incluso, en alguno, hasta abocado al fracaso. Así, pues, la experiencia, en este instante, parece estar muy lejos de extenderse al resto de los institutos marroquíes. Asimismo, se venía gestando un nuevo proyecto que prometía estar en funcionamiento a partir del curso 2005-06, y que pretende extender el español también a la Preparatoria marroquí, y así adelantar el contacto con la segunda lengua extranjera. Si bien, en lo que se refiere a los programas por los que se ha de regir esta enseñanza y a los materiales didácticos que se han de programar para este fin, quizá sorprenda un poco decir que, sobre estos aspectos tan primordiales para un proyecto de semejante envergadura, -literalmente- "no hay nada escrito", ya que todo cuanto consta en la nueva disposición es que se adoptaría un procedimiento oral, dejando total libertad tanto en materia de organización como de elección de materiales al profesor $^{30}$.

Por otra parte, el plan curricular del español, desde el curso 97-98, se fundamenta en dos documentos pedagógicos esenciales, uno de los cuales, el Diseño Curricular del Español, fue especialmente concebido para la Opción de Lengua Española de esa improvisada Sección de Lenguas, mientras que el de las Orientaciones Pedagógicas (O.P.E) tiene carácter general. Ambos documentos fueron obra de un equipo pedagógico de profesores e inspectores del español, con la colaboración de la Asesoría Linguiística de la Consejería de Educación, quienes contemplaron básicamente la experiencia de los largos años de docencia dominada por una visión conservadora y prescriptiva, para a partir de ahí, y gracias a esos documentos, ir avanzando hacia un enfoque metodológico más sintetizador y ecléctico ${ }^{31}$.

\section{El panorama actual}

En la actualidad, se estima que alrededor de tres millones de marroquíes hablan el español correctamente, y dos millones más lo conocen ${ }^{32}$. Existen, asimismo, diez Centros de Enseñanza españoles distribuidos entre Casablanca, Rabat, Larache, Tánger, Tetuán, Alhucemas y Nador, entre Colegios de EGB, Institutos de Bachillerato, y centros de Formación Profesional, además de cinco -de los nueve existentes en todo el mundo árabe- centros del Instituto Cervantes.

${ }^{29}$ Santamaría (2000: 230).

${ }^{30} \mathrm{Si}$ bien tenemos constancia de que la Editorial Anaya está realizando gestiones y reuniones con profesores e inspectores marroquíes para intentar implantar el manual Mañana con sus tres correspondientes niveles en la Preparatoria marroquí.

${ }^{31}$ Santamaría (2000: 231).

32 Tomo el dato de la Entrevista a D. J. Garre Navarro, director del Instituto Severo Ochoa en Tánger en Aoufi (2001): http://perso.menara.ma/ a.aoufi/14_index.HTM. 
Según las estadísticas de la Consejería de Educación correspondientes al año $2003^{33}$, el número de alumnos que cursan estudios de español en institutos marroquíes de secundaria, asciende a 48.587, -cifra nada desdeñable, pero lejos de alcanzar el objetivo de generalizar el español en la secundaria-, distribuidos entre 236 liceos. Se encargan de impartirles el español unos 600 profesores, supervisados por 29 Inspectores marroquíes, los cuales colaboran con la Consejería de educación. La demanda del español es cada vez mayor, y el español es, cada vez más, la opción predilecta entre los alumnos, aunque siempre después del inglés.

Tabla 1. Enseñanza de lenguas extranjeras en la secundaria marroquí (2000-2001)

\begin{tabular}{lccc}
\hline Lengua & $N^{o}$. de clases & $N^{o}$ de alumnos & $N^{o}$ de profesores \\
\hline Inglés & 12.837 & 389.925 & 3.440 \\
Español & 1.723 & 36.839 & 598 \\
Alemán & 1.073 & 7.198 & 143 \\
Italiano & 66 & 1.692 & 34 \\
& & & \\
\hline
\end{tabular}

Tabla 2. Enseñanza de lenguas extranjeras en la secundaria marroquí (2001-2002)

\begin{tabular}{lccc}
\hline Lengua & $N^{o}$. de clases & $N^{o}$ de alumnos & $N^{\circ}$ de profesores \\
\hline Inglés & 16.493 & 425.574 & 3.420 \\
Español & 1.467 & 44.274 & 571 \\
Alemán & 280 & 8.146 & 119 \\
Italiano & 66 & 1.864 & 33 \\
& & & \\
\hline
\end{tabular}

Tabla $3^{34}$. Enseñanza de lenguas extranjeras en la secundaria marroquí (2002-2003)

\begin{tabular}{lccc}
\hline Lengua & $N^{o}$. de clases & $N^{o}$ de alumnos & $N^{o}$ de profesores \\
\hline Inglés & 13.517 & 475.275 & 3.441 \\
Español & 1.601 & 50.965 & 591 \\
Alemán & 268 & 8.050 & 123 \\
Italiano & 64 & 1.904 & 35 \\
& & & \\
\hline
\end{tabular}

${ }^{33}$ Vacas Lobato (2003): http://sedll.org/doc-es/publicaciones/glosas/nmon10/marruecos.pdf

${ }^{34}$ Estos datos son d el Instituto Cervantes y están publicados en: http://cvc.cervantes.es/obref/anuario/ anuario_03/munoz/p03.htm 
No obstante, y pese al crecimiento de la demanda en este sector, no es posible hacer pronósticos, puesto que la política estatal, muchas veces, condicionada por las dificultades económicas, se traduce en la aplicación de medidas restrictivas que -directa o indirectamenterepercuten en el terreno educativo. El Estado, por falta de presupuesto, recurre habitualmente a la estrategia de cerrar algunas de las secciones de las Escuelas Normales Superiores (ENS), las cuales constituyen la salida profesional más ambicionada por los titulados universitarios, y la del español evidentemente no constituye ninguna excepción, y así, según la oferta del Estado, se procede a la suspensión o a la apertura de una convocatoria para seleccionar futuros profesores de español, mediante una oposición a la que acceden, en su mayoría, licenciados en Filología Hispánica, y que una vez superada esa prueba, reciben un Curso de Formación que ofertan esos centros con la duración de un año, al cabo del cual se convierten en profesores de español.

La enseñanza en los institutos marroquíes, sin embargo, adolece de muchas dificultades y se lleva a cabo en unas condiciones muy precarias. Los escasos medios o recursos dedicados al sector de enseñanza en general apenas pueden proporcionar un material elemental, poco menos que rudimentario en lo que a centros e instalaciones se refiere. Y en cuanto al material didáctico, no existe apenas la posibilidad de utilizar otro que no sea los manuales oficiales $^{35}$, de elaboración nacional, editados por el Ministerio de Educación -y cuyos autores son profesores marroquíes-, con sus correspondientes niveles $1^{\circ}, 2^{\circ}$ y $3^{\circ}$, que desde los años noventa, han venido a sustituir a los anteriores de impronta y "confección" francesa. Quizá sea un dato positivo señalar que, en la última década, se están revisando por la Asesoría Lingüística integrada en la Consejería de Educación. Pese a los inconvenientes que puedan presentar o a la inadecuación de algunos contenidos -que necesitan de una actualización-, resultan bastante mejores que los anteriores y que procuran, en la medida de lo posible, integrar el componente sociocultural ofreciendo una visión menos tópica y que se acerca bastante a la real, mediante la inclusión de un material auténtico y variado, eso sí, de carácter eminentemente literario. Sin embargo, quizá sea todavía presuntuoso, o un poco fuera de lugar, el pensar, en el marco de las circunstancias actuales, en alternativas como el uso de un material informático o interactivo, ya que el profesor, muy mal remunerado -por cierto,- se encuentra con la ardua tarea de tener que ingeniárselas todos los días, para enseñar, desde sus modestas posibilidades, a unos alumnos, las más de las veces, totalmente ajenos al mundo de lo español, una lengua y una cultura que probablemente no conseguirá nunca despertar su interés o motivación.

La consejería, a este fin, organiza permanentemente cursos de formación y de actualización didáctica dedicados especialmente a los profesores de español, para que éstos incorporen en su método las últimas técnicas de la enseñanza de las lenguas extranjeras. Asimismo, colabora en la elaboración de publicaciones periódicas y de material didáctico complementario, pero cabe señalar que esta estrategia apenas surte efecto y que ese material apenas es aprovechado en las aulas marroquíes, debido a restricciones administrativas que obligan al profesor a ajustarse al programa estableciéndole plazos para la ejecución del plan curricular.

La línea de actuación española en materia educativa en Marruecos, tiene su cauce en el Instituto Cervantes dependiente del MAE, o en la Consejería de Educación aneja a la Emba-

${ }^{35}$ Santamaría (2000: 229). 
jada Española en Rabat, encargada de la gestión y financiación de los diez centros españoles existentes en el país. Hay que decir que la enseñanza del español en esos centros goza de unas excelentes condiciones, ya que se ha podido beneficiar de las más actuales investigaciones en materia didáctica. Sin embargo, esas instituciones funcionan independientemente del sistema educativo oficial, y los centros que gestionan siguen en sus currículos al sistema educativo español, y en ese sentido, exceptuando la todavía tímida línea de cooperación que se ha abierto recientemente entre esas entidades -a través de la Asesoría lingüística básicamentey las autoridades marroquíes, no se producen muchos intercambios ni mayor relación, y el español en los institutos marroquíes, obviamente, apenas se está beneficiando de esa experiencia positiva y de la metodología seguida en esos centros.

No obstante, el panorama es alentador, y el español es cada vez más un idioma "conocido", sobre todo al sur de Marruecos, donde se desconocía por completo, y se excluía -a menos que el exceso de demanda sobre el inglés no le dejara lugar al alumno a elegir, por falta de plazas-. Es cada vez una opción más preferida y las estadísticas refutan la teoría de que se trate de un fenómeno debido únicamente a las mencionadas circunstancias administrativas, ya que el número de alumnos de español dentro del sistema educativo marroquí se ha quintuplicado por lo menos, en los últimos veinticinco años ${ }^{36}$.

La universidad supone otra faceta de esa creciente expansión del español en Marruecos. En las universidades marroquíes, de la misma manera que en los institutos, se asiste a una demanda cada vez mayor del español, a la que ha acompañado la apertura de nuevos departamentos de Lengua Española. Desde que se inauguró el primer Departamento en Rabat -de muy reducida afluencia y actividad- hasta hoy, el panorama en las universidades marroquíes ha cambiado bastante, y cinco de las mismas ya cuentan con un Departamento de Filología Española $^{37}$, cuyas matrículas ascienden a 2600 alumnos $^{38}$ y alguno, como el de Tetuán o Rabat, hasta ofrece la posibilidad de cursar Estudios de Tercer Ciclo, si bien la investigación se encuentra muy restringida y los cursos de doctorado, debido a problemas de organización interna, no se ofertan todos los años. Asimismo, en los demás departamentos, el español figura como segunda lengua optativa a elegir entre otras como el inglés, el italiano, el hebreo o el persa, además de aparecer como lengua complementaria o de apoyo en algunas Escuelas Superiores, pero siempre con carácter optativo.

Como fruto de la política cultural española desplegada asiduamente en las 'últimas décadas, el español ha venido a ostentar un puesto, cuando menos, destacado entre los demás idiomas, y aunque, sin dificultades, ha podido afianzarse con más propiedad y ha conseguido despertar el interés de un colectivo marroquí bastante importante. De hecho, existen varias asociaciones de hispanistas marroquíes, constituidas -en su mayoría- de profesores y escritores marroquíes de expresión española, entre las que destacaríamos la de AHISMA y AEMLE, que celebran, periódicamente, encuentros y coloquios que culminan con algunas publicaciones.

La presencia del español en los medios de comunicación, sin embargo, todavía es muy tímida: sólo se emite un telediario en español que no es, en su esencia, más que una traduc-

${ }^{36}$ Véase Aoufi (2001): http://perso.menara.ma/ a.aoufi/14_index.HTM.

${ }^{37}$ A saber, Rabat, Fez, Tetuán y Casablanca.

38 Vacas Lobato (2003): http://sedll.org/doc-es/publicaciones/glosas/nmon10/marruecos.pdf 
ción literal de las noticias nacionales, ya emitidas a lo largo del día, si acaso con alguna referencia final y muy breve a alguna actividad que se haya celebrado en Marruecos relacionada con España.

En cuanto a la prensa, existen periódicos que consagran alguna sección a artículos en español, o incluso alguno íntegramente en español como la Mañana del Sáhara y el Magreb, pero que, evidentemente, tienen una difusión muy local.

A todo ello hay que añadir la importante herencia española que constituye un signo diferencial de la que fue Zona del Protectorado español con respecto a la del Protectorado francés, y que deja vislumbrarse constantemente, e independientemente de las políticas cursadas, una enorme influencia que se aprecia en el habla local, salpicada por todo tipo de hispanismos "arabizados", en los detalles de las calles que conservan todavía nombres españoles, o en los menús de los restaurantes escritos en español.

Sin embargo, esta realidad, que pudo haber sido muy ventajosa para el destino del español en Marruecos, por los diferentes motivos aquí expuestos, no fue explotada de la forma más propicia, y fue el francés, en cambio, quien pudo prevalecer, por políticas de fomento y promoción francesas y evidente apoyo interno por parte de las autoridades marroquíes, y en segundo lugar el inglés, ya no por razones históricas ni geográficas, sino por ser una lengua de interés e imposición internacional.

El español, lengua en constante expansión, a pesar de todo, y quieran que no las políticas, es cada vez más una lengua de interés en Marruecos y que acapara mayor número de candidatos. Esta realidad se traduce, de forma patente, en las aulas marroquíes que acogen, cada vez, un mayor número de alumnos de español. Estas aulas constituyen, las más de las veces, el primer contacto del alumno marroquí con lo español, y por supuesto el primer eslabón de una potencial carrera en español. Indagar sobre las formas de desarrollo de esta enseñanza en el seno del sistema educativo oficial y sondear las dificultades que encuentra el alumno marroquí a la hora de emprender el "viaje al español", partiendo de la base del árabe, y de la de la segunda lengua obligatoria -que supone ser el francés-, constituye el principal objetivo de esta investigación.

\section{Bibliografía}

Aoufi, Abdelhak (coord.) (2001). "Entrevista a D. J. Garre Navarro, director del Instituto Severo Ochoa en Tánger", Babel, $\mathrm{n}^{\circ}$ 14: http://perso.menara.ma/ a.aoufi/14 index.HTM

Bennani, Aziza (1996). "Relaciones culturales y cooperación científica hispano-marroquíes: un balance de cuarenta años después de la independencia del Magreb" en Awráq. Estudios sobre el mundo árabe e islámico contemporáneo, volumen XVII. Madrid: ICMA, 283291.

Chouiref, Moustapha (1991). "Primeros resultados de una encuesta sobre el estado actual de la enseñanza del español en Marruecos”, en la Revista Marroquí de Estudios Hispánicos (Semestral), 2: 107- 115.

Convenio De Cooperación Cultural entre el Gobierno De España y el Gobierno del reino De Marruecos, 8 de noviembre de 1979, Madrid.

Fernández Suzor, Cecilia (1992). "Las relaciones culturales"hispano-marroquíes en la perspectiva de los noventa" en Bernabé López (coord.). España-Magreb, siglo XXI. El porvenir de una vecindad. Madrid: Mapfre, 327- 335. 
Fernández Suzor, Cecilia (1993). "Los centros culturales y el Instituto Cervantes en Marruecos" en V. Morales Lezcano (coord.) Presencia cultural de España en el Magreb: pasado y presente de una relación cultural 'sui generis' entre vecinos mediterráneos. Madrid: MAPFRE, 169-174.

García Figueras, Tomás (1940). Notas sobre instrucción y cultura en Marruecos (hasta 1935), Alta comisaría de España en Marruecos. Tetuán.: Editora Marroquí.

Loh, Amina (1968). La enseñanza primaria en el norte de Marruecos durante la primera mitad del siglo XX, Madrid: Universidad Central de Madrid.

Lourido Díaz, R. (1993). "La Iglesia española en el Magreb y sus aportaciones culturales" en Morales Lezcano, V. (coord.), Presencia cultural de España en el Magreb: pasado y presente de una relación cultural sui generis entre vecinos mediterráneos. Madrid: Mapfre, 47-73.

Ruiz Orsatti, Ricardo (1918). La enseñanza en Marrueco. Tetuán: La Papelera Africana.

Ministère de 1’Enseignement Primaire et Secondaire (1976). Programme d'espagnol, instructions officielles. Setiembre de 1976. Rabat.

Moratinos, Miguel Ángel (1993). "Presencia cultural de España en el Magreb" en Morales Lezcano, V. (coord.), Presencia cultural de España en el Magreb: pasado y presente de una relación cultural sui generis entre vecinos mediterráneos. Madrid: Mapfre, 175- 194.

Naji, Jamal Eddine (2000). "L’image de l’Espagne dans les médias marocains" en el II Seminario hispano-marroquí: aprender a conocerse percepciones sociales y culturales entre España y Marruecos, Fundación Hassan II para los marroquíes residentes en el extranjero. Rabat: Fundación Repsol YPF, 157- 168.

País (El) Setiembre de1989. Madrid.

Pino (del), Domingo (1990). Marruecos entre la tradición y el modernismo, Granada: Universidad de Granada.

Romero López, Jesús. Algunos retazos sobre la educación no universitaria en Marruecos, http:/ /members.es.tripod.dekasbah01/educacion marroqui.htm

Salas Larrazábal, R. (1992). El protectorado de España en Marruecos. Madrid: Mapfre.

Santamaría, Eduardo (2000). "El juego de la poesía en Marruecos. Una aproximación a la realidad de la enseñanza del español como lengua extranjera", en A. Martinez et al.: Enseñanza de la lengua. Granada: Universidad de Granada, 227- 246.

Tanouti, M./ Muati, F. (1997). El currículo del español como Lengua Extranjera en la secundaria marroquí y en el Instituto Cervantes, memoria final del Curso de Formación de la E.N.S de Tetuán (inédita). Tetuán.

Vacas Lobato, Teresa (2003). "El español en el mundo. La enseñanza del español en Marruecos" en Glosas Didácticas. Revista electrónica internacional, ISSN 1576-7809, № 10, Consejería de Educación en Marruecos.

Valderrama Martínez, Fernando (1956). Historia de la acción cultural de España en Marruecos (1912-1956), Alta Comisaría de España en Marruecos. Tetuán: Editora Marroquí.

Valderrama Martínez, Fernando (1954). Temas de educación y cultura en Marruecos. Tetuán: Editora Marroquí. 